\title{
An XMM-Newton study of the X-ray binary MXB 1659-298 and the discovery of narrow $\mathrm{X}$-ray absorption lines
}

\begin{abstract}
L. Sidoli, T. Oosterbroek, A. N. Parmar, D. Lumb, and C. Erd
Space Science Department of ESA, ESTEC, Postbus 299, 2200 AG Noordwijk, The Netherlands

Received 23 July 2001 / Accepted 17 September 2001

Abstract. We report the discovery of narrow X-ray absorption lines from the low-mass X-ray binary MXB 1659298 during an XMM-Newton observation in 2001 February. The $7.1 \mathrm{hr}$ orbital cycle is clearly evident with narrow X-ray eclipses preceded by intense dipping activity. A sinusoid-like OM $B$-band modulation with a peak-to-peak modulation of $\sim 0.5 \mathrm{mag}$ and a minimum coincident with the X-ray eclipse is visible. EPIC and RGS spectra reveal the presence of narrow resonant absorption features identified with O VIII $1 \mathrm{~s}-2 \mathrm{p}, 1 \mathrm{~s}-3 \mathrm{p}$ and $1 \mathrm{~s}-4 \mathrm{p}$, Ne X $1 \mathrm{~s}-2 \mathrm{p}$, Fe Xxv 1s-2p, and Fe Xxvi 1s-2p transitions, together with a broad Fe emission feature at $6.47_{-0.14}^{+0.18} \mathrm{keV}$. The equivalent widths of the Fe absorption features show no obvious dependence on orbital phase, even during dipping intervals. The equivalent widths of the other features are consistent with having the same values during persistent and dipping intervals. Previously, the only X-ray binaries known to exhibit narrow X-ray absorption lines were two superluminal jet sources and it had been suggested that these features are related to the jet formation mechanism. This now appears unlikely, and instead their presence may be related to the viewing angle of the system. The MXB 1659-298 0.6-12 keV continuum is modeled using absorbed cutoff power-law and blackbody components. During dips the blackbody is more strongly absorbed than the power-law. The spectral shape of the $3.6 \%$ of 0.5-10 keV emission that remains during eclipses is consistent with that during non-dipping intervals.
\end{abstract}

Key words. accretion, accretion disks - stars: individual: MXB 1659-298 - stars: neutron - X-rays: general

\section{Introduction}

MXB 1659-298 is a transient bursting X-ray source discovered by SAS-3 (Lewin et al. 1976). Observations with SAS-3 and HEAO-1 detected a clearly modulated persistent emission which showed erratic intensity variations followed by $15 \mathrm{~min}$ duration X-ray eclipses which repeated periodically (Cominsky \& Wood 1984). By combining data spanning $\sim 2$ years an orbital period of $7.11 \mathrm{~h}$ was obtained (Cominsky \& Wood 1989). Following the discovery using EXOSAT of a second bursting, dipping, eclipsing, transient (EXO 0748-676; Parmar et al. 1986) the region of sky containing MXB 1659-298 was observed by EXOSAT. However, the source was not present. Indeed, until recently MXB 1659-298 has not been observed to be active. However, on 1999 April 2, after a 21 year quiescent interval, an $18 \mathrm{~m}$ Crab (2-9 keV) source at a position consistent with MXB 1659-298 was detected in one of the BeppoSAX Wide Field Cameras (in 't Zand et al. 1999). Observations a day later confirmed that the $V=18.3$ optical counterpart to MXB 1659-298 (V2134 Oph; Doxsey et al. 1979) had brightened significantly and was exhibiting high excitation He II emission, confirming that the source was undergoing an outburst (Augusteijn et al. 1999).

Send offprint requests to: L. Sidoli,

e-mail: sidoli@ifctr.mi.cnr.it
MXB 1659-298 was observed by RXTE a few days later (Wachter et al. 2000) confirming that the source had resumed its strong and persistent X-ray emission. Contemporaneous observations revealed that the optical emission exhibits a 0.2 mag eclipse feature coincident with the X-ray eclipse. The $0.5-30 \mathrm{keV}$ BeppoSAX spectrum obtained in 2000 August was modeled by a combination of a soft disk-blackbody and a harder Comptonized component (Oosterbroek et al. 2001). The residuals to this fit suggest the presence of emission features due to Ne-K/Fe-L and Fe-K in the spectrum.

Only two Low-Mass X-ray binary (LMXRB) systems are known that exhibit both dips and eclipses (about 10 exhibit only dips). During dips the observed spectral changes are inconsistent with simple absorption from cold material, as might be expected. Detailed modeling of these spectral changes provides a powerful means of studying the structure and location of the emitting and absorbing regions in LMXRB (e.g., Parmar et al. 1986; Church \& Bałucińska-Church 1995; Church et al. 1997). This modeling has revealed that at least two emission components are required which appear to undergo different amounts of absorption during dips. In the case of EXO 0748-676, (the other eclipsing and dipping LMXRB), recent XMM-Newton Reflection Grating Spectrometer (RGS) observations have revealed the presence of discrete 
Table 1. XMM-Newton instrument configurations.

\begin{tabular}{lccl}
\hline Instrument & $\begin{array}{c}\text { Energy } \\
\text { Range } \\
(\mathrm{keV})\end{array}$ & $\begin{array}{c}\text { Net } \\
\text { Exposure } \\
(\mathrm{ks})\end{array}$ & Mode \\
\hline EPIC PN & $0.2-15$ & 21.5 & Small window \\
EPIC MOS1 & $0.2-15$ & 30.0 & Timing \\
EPIC MOS2 & $0.2-15$ & 30.0 & Full window \\
RGS & $0.35-2.0$ & 31.1 & Spectroscopy $+Q$ \\
OM & $\ldots$ & $20 \times 1$ & $B$ filter \\
\hline
\end{tabular}

structures due to absorption and emission from ionized Ne, O and N (Cottam et al. 2001). Simultaneous European Photon Imaging Camera (EPIC) data revealed that the 0.2-10 keV spectra can be fit with a two component model consisting of a central Comptonized component and a more extended thermal halo (Bonnet-Bidaud et al. 2001). The spectral variations observed during dips were mainly accounted for by variations in the absorbing column affecting the Comptonized component only.

\section{Observations and data analysis}

The XMM-Newton Observatory (Jansen et al. 2001) includes three $1500 \mathrm{~cm}^{2} \mathrm{X}$-ray telescopes each with an EPIC at the focus. Behind two of the telescopes there are RGS arrays (den Herder et al. 2001). In addition, a coaligned optical/UV monitor telescope (OM, Mason et al. 2001) is included as part of the payload. Two of the EPIC imaging spectrometers use MOS type CCDs (Turner et al. 2001) and one uses a PN type CCD (Strüder et al. 2001).

The data analysis reported here used event lists produced by the standard XMM-Newton SAS (Science Analysis Software, V.5.0.0) EMPROC, EPPROC and RGSPROC tasks which were further filtered using XMMSELECT V.2.35.4. For the PN only pattern 0 (single pixel) events were selected. Known hot, or flickering, pixels and electronic noise were rejected using the SAS and the low-energy cutoff was set to $0.2 \mathrm{keV}$. For the MOS, Xray events corresponding to patterns $0-12$ were selected.

Source counts were extracted from circular regions of $40^{\prime \prime}$ radius centered on MXB 1659-298 for the PN instrument. Background counts were obtained from similar regions offset from the source position. RGS source and background events were extracted by making spatial and energy selections on the event files. Wavelengths were then assigned to the dispersion coordinates using the latest calibration parameters. In order to ensure applicability of the $\chi^{2}$ statistic, extracted spectra were rebinned such that at least 20 counts per bin were present and such that the energy resolution was not oversampled by more than a factor 3. In order to account for systematic effects a $2 \%$ uncertainty was added quadratically to each spectral bin. The photo-electric absorption cross sections of Morrison \& McCammon (1983), $\sigma_{\mathrm{MM}}$, are used throughout. All spectral uncertainties and upper-limits are given at $90 \%$ confidence. Quoted count rates are corrected for instrumental deadtime, whereas lightcurves are not.

XMM-Newton observed the region of sky containing MXB 1659-298 as a Target of Opportunity (TOO) between 2000 March 21 23:55 and March 22 07:16 UTC. Unfortunately, the observation was curtailed for technical reasons. In addition, much of the data that were obtained was not correctly transmitted to the XMM-Newton data center at Vilspa. Currently, these data have not been recovered. An examination of the $6.7 \mathrm{ks}$ of PN exposure that is available shows that MXB 1659-298 is clearly detected undergoing dipping, an eclipse and an interval of "persistent" emission. However, an examination of the PN spectrum shows that the instrument configuration was abnormal and we await an updated calibration before performing a spectral analysis of this data. Thus, in this paper we present only results from a second observation in 2001 February.

Due to the problems encountered in the first observation, the region on sky containing MXB 1659-298 was re-observed by XMM-Newton between 2001 February 20 08:28 and 16:52 UTC. Table 1 provides details of the instrument modes used. The thin filter was used with the EPIC CCDs. The mean 2-10 keV flux obtained from the $\mathrm{PN}$ corresponds to a luminosity of $1.6 \times 10^{37} \mathrm{erg} \mathrm{s}^{-1}$ for a distance of $15 \mathrm{kpc}$ (Cominsky 1981).

\section{EPIC results}

\subsection{X-ray lightcurve}

The $0.5-10 \mathrm{keV}$ MXB 1659-298 background subtracted light curve obtained from the $\mathrm{PN}$ during the 2001 February observation is shown in Fig. 1 with a binning of $32 \mathrm{~s}$. At the start of the observation the source was undergoing an interval of rapid dipping activity, prior to an eclipse between 2001 February 20 09:10 and 09:25. After the first egress the source displayed a long interval of "persistent" emission punctuated by an X-ray burst at 2001 February $2010: 12 \mathrm{hr}$. The height of the burst is strongly reduced by the binning used in Fig. 1. Fitting the burst spectrum with a blackbody, after the subtraction of the contribution from the persistent emission before the burst, gives a temperature of $1.4_{-0.1}^{+0.2} \mathrm{keV}$ and a radius of $15_{-5}^{+7} \mathrm{~km}$. Following an interval of $\sim 6 \mathrm{hr}$, a second eclipse is visible. After the second eclipse there is a short, deep, dip just prior to the end of the observation. In the $0.5-$ $10 \mathrm{keV}$ energy range the first and second eclipse ingresses take $15.2 \pm 1.1 \mathrm{~s}$ and $25.1 \pm 1.1 \mathrm{~s}$ (at $68 \%$ confidence), respectively. Similarly the egresses takes $14.4 \pm 0.8 \mathrm{~s}$ and $30.4 \pm 1.0 \mathrm{~s}$. The dips are deep with the $2-10 \mathrm{keV}$ flux often reaching the eclipses level of emission. The upper panel of Fig. 1 shows the PN hardness ratio (counts in the energy range $2.5-10 \mathrm{keV}$ divided by those between $0.5-2.5 \mathrm{keV}$ ) with a binning of $128 \mathrm{~s}$. During the dips, which occur between orbital phases $\sim 0.7-1.0$, the source becomes harder than during the persistent emission. Such behavior is a common feature of LMXRB dips. 


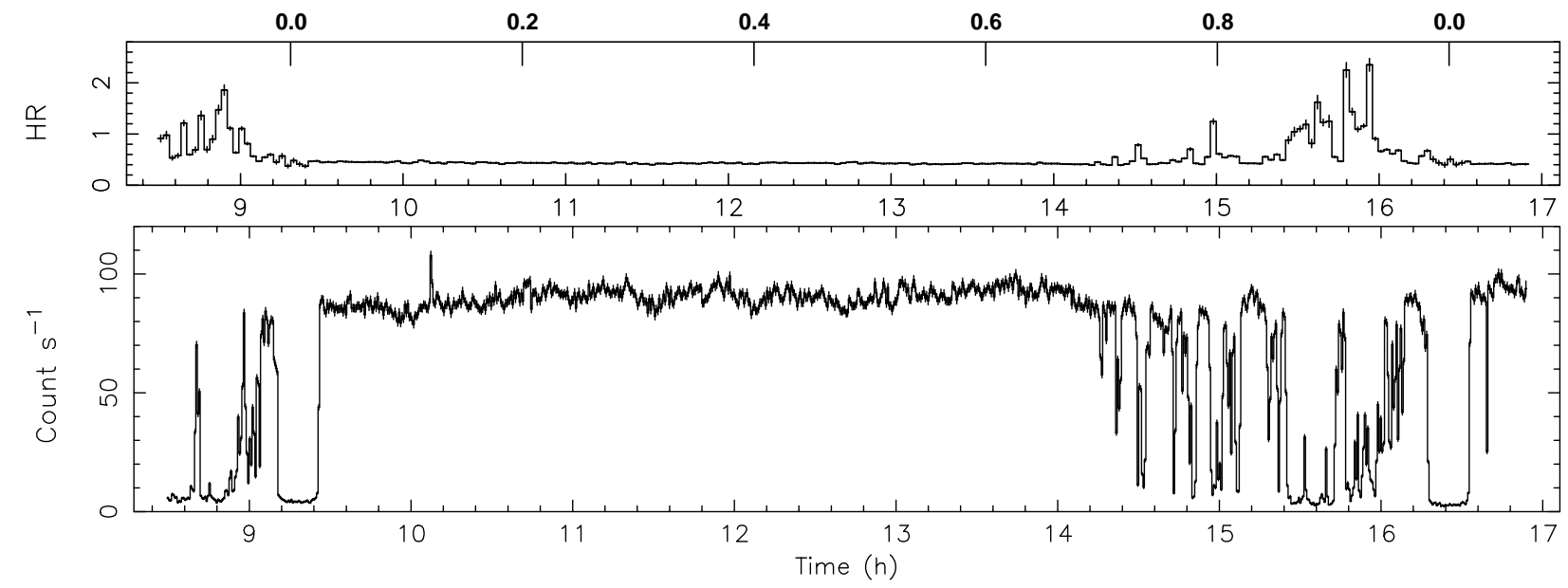

Fig. 1. The XMM-Newton PN 0.5-10 keV light curve of MXB 1659-298 with a binning of 32 s. Time is hours of 2001 February 20. Two eclipses, periods of intense dipping activity and a burst at approximately $10.1 \mathrm{hrs}$ are visible. The upper panel shows the $\mathrm{PN}$ hardness ratio, $\mathrm{HR}$, (counts in the $2.5-10 \mathrm{keV}$ energy range divided by those between $0.5-2.5 \mathrm{keV}$ ) with a binning of $128 \mathrm{~s}$. Orbital phase is indicated above.
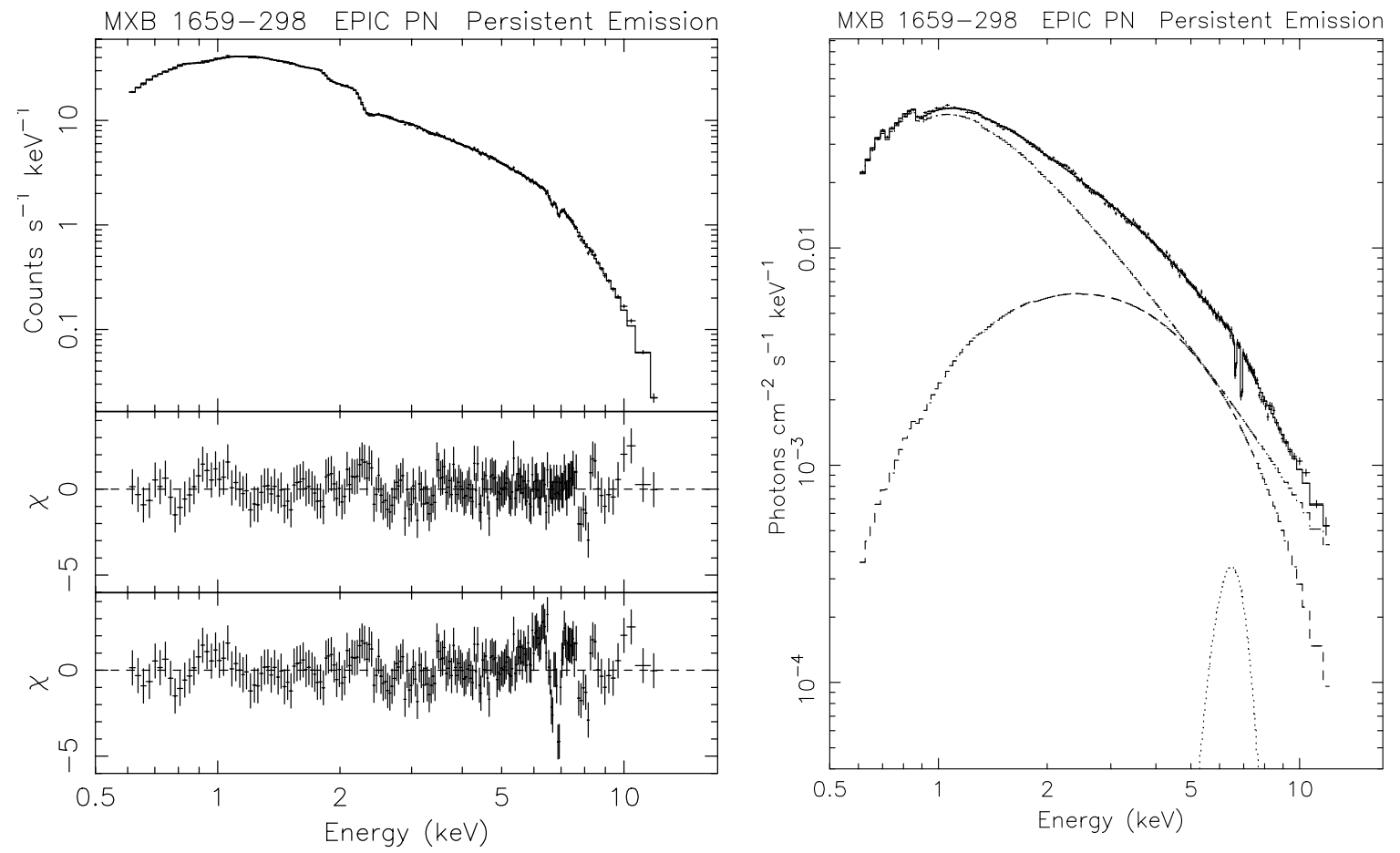

Fig. 2. The 0.6-12 keV PN persistent emission. The left panels show the best-fit count spectrum and the residuals. The lowest left panel shows the residuals when the normalizations of the narrow absorption and broad emission features are set to zero. The structured residuals between $7.0-10.0 \mathrm{keV}$, as well as the positive residuals around $2.2 \mathrm{keV}$ are consistent with being due to instrumental effects. The right panel shows the best-fit deconvolved photon spectrum with the individual components indicated. The cutoff power-law dominates $\gtrsim 5 \mathrm{keV}$.

\subsection{Persistent emission spectrum}

The persistent emission spectrum during the 2001 February observation was investigated by extracting the $0.2-12 \mathrm{keV}$ PN events obtained between 2001 February 20 09:30 and 14:00 UTC, which is an interval free of eclipses and obvious dipping activity. Times corresponding to the X-ray burst around 2001 February 20 10:12 hrs were also excluded. This selection results in a
0.6-12 keV count rate of $125.0 \pm 0.1 \mathrm{~s}^{-1}$ with an exposure time of $12.0 \mathrm{ks}$. In the PN small window mode only a $63 \times 64$ pixel region of the central CCD is read out with a time resolution of $5.7 \mathrm{~ms}$ in order to reduce the effects of pile-up. This is expected to be significant at count rates of $\gtrsim 100 \mathrm{~s}^{-1}$, comparable to that of the persistent emission (Strüder et al. 2001). The effects of pile-up were investigated by extracting events in an annulus outside the core of the MXB 1659-298 point spread 
function (PSF) and comparing their spectrum with that obtained above. As expected, this showed that the overall spectrum is affected by pile-up. To minimize this, events from the inner $6^{\prime \prime}$ radius core of the PSF were excluded from the PN spectrum which is used in the subsequent analysis. This leaves a background subtracted count rate of $78.90 \pm 0.08 \mathrm{~s}^{-1}$.

Unfortunately, it is not yet possible to analyze MOS Timing Mode data. The MOS2 Full Window mode data is strongly affected by pile-up and a spectrum of the events in the wings of the PSF was extracted. This had a count rate of only $6.16 \pm 0.02 \mathrm{~s}^{-1}$. A comparison with the PN spectrum showed good agreement between the two, except at energies $\lesssim 0.6 \mathrm{keV}$. A further comparison with the RGS continuum (see Sect. 4.1) showed good agreement between the MOS and RGS spectra and so PN data below $0.6 \mathrm{keV}$ were excluded. Following discussion with the XMM-Newton Science Operations Center personnel, this difference is attributed to residual calibration uncertainties in the PN small window mode.

We first tried to fit the persistent $0.6-12 \mathrm{keV}$ PN spectrum of MXB 1659-298 with simple models such as an absorbed power-law, cutoff power-law $\left(E^{-\alpha} \exp -\left(E_{\mathrm{c}} / k T\right)\right)$, thermal bremsstrahlung, blackbody, and multicolor diskblackbody (Mitsuda et al. 1984; Makishima et al. 1986), but none of these provided acceptable fits with values of reduced $\chi^{2}>2$ and large and structured residuals in all cases. Comptonized emission models, such as the COMPST and COMPTT models in XSPEC (see e.g., Titarchuk 1994), were the only single-component models providing reduced $\chi^{2}$ values of $<2$, although significant residuals still remained at high energies. Indeed, the unacceptable fits can be mostly attributed to the presence of narrow negative residuals around 6.65 and $6.90 \mathrm{keV}$, suggesting the presence of discrete absorption features. Examination of the PN background spectra does not reveal the presence of features at these energies suggesting that they are not due to improper background subtraction.

Next, we tried to fit the persistent spectrum with different models, always including the two Gaussian absorption lines at energies of $\sim 6.65 \mathrm{keV}$ and at $\sim 6.90 \mathrm{keV}$. Cutoff power-law, COMPST and COMPTT models were tested, giving $\chi^{2}$ values of 724.2 for 253 degrees of freedom (d.o.f.), 307.3 for 253 d.o.f. and 283.8 for 252 d.o.f., respectively. In all the above fits, the presence of broad positive residuals around $6 \mathrm{keV}$ suggests the presence of an additional Gaussian line, but this time in emission. This reduced the $\chi^{2}$ to 302.5 for 250 d.o.f., to 282.3 for 250 d.o.f. and to 264.3 for 249 d.o.f. for the cutoff power-law, the COMPST and the COMPTT continuum models, respectively. In all three models, this additional Gaussian line is significant at a confidence level of $>99.99 \%$. Inspection of the residuals shows that, in the case of the cutoff power-law fit, broad structured residuals $\lesssim 2 \mathrm{keV}$ are still present, suggesting the need for an additional continuum component. Blackbody and multicolor disk-blackbody were added to the above continuum and line models and the fits repeated. The inclusion of these low-energy components to
Table 2. Best-fit spectral parameters for the persistent MXB 1659-298 emission with a cutoff power-law, with photon index $\alpha$ and cutoff energy $E_{\mathrm{c}}$, and a blackbody, with temperature $k T_{\mathrm{bb}}$ and radius $R_{\mathrm{bb}}$ together with 2 Gaussian features in absorption and one in emission. $E_{\text {line }}$ is the line centroid, $\sigma$ its width, $E W$ the equivalent width, and $I_{\text {line }}$ the intensity. Uncertainties are given at $90 \%$ confidence for one interesting parameter, except for upper-limits which are $68 \%$ confidence. The blackbody radius and normalization have been corrected for the missing flux when extraction annuli were used and assume a distance of $15 \mathrm{kpc}$.

\begin{tabular}{|c|c|c|}
\hline Component & Parameter & Value \\
\hline \multirow{4}{*}{$\begin{array}{l}\text { Cutoff } \\
\text { power-law }\end{array}$} & $N_{\mathrm{H}}\left(10^{22}\right.$ atom $\left.\mathrm{cm}^{-2}\right)$ & $0.35_{-0.01}^{+0.02}$ \\
\hline & $\alpha$ & $1.90 \pm 0.02$ \\
\hline & $E_{\mathrm{c}}(\mathrm{keV})$ & $15_{-4}^{+29}$ \\
\hline & Norm (ph. $\mathrm{keV}^{-1} \mathrm{~cm}^{-2} \mathrm{~s}^{-1}$ ) & $0.168_{-0.005}^{+0.006}$ \\
\hline \multirow[t]{2}{*}{ Blackbody } & $k T_{\mathrm{bb}}(\mathrm{keV})$ & $1.30_{-0.06}^{+0.01}$ \\
\hline & $R_{\mathrm{bb}}(\mathrm{km})$ & $4.8_{-0.03}^{+0.07}$ \\
\hline Fe emission & $E_{\text {line }}(\mathrm{keV})$ & $6.47_{-0.14}^{+0.18}$ \\
\hline \multirow[t]{3}{*}{ feature } & $\sigma(\mathrm{keV})$ & $0.60_{-0.16}^{+0.12}$ \\
\hline & $I_{\text {line }}\left(10^{-4} \mathrm{ph} \mathrm{cm}^{-2} \mathrm{~s}^{-1}\right)$ & $5.6_{-1.6}^{+1.9}$ \\
\hline & $E W(\mathrm{eV})$ & $160_{-40}^{+60}$ \\
\hline Fe XXV & $E_{\text {line }}(\mathrm{keV})$ & $6.64 \pm 0.02$ \\
\hline absorption & $\sigma(\mathrm{keV})$ & $<0.063$ \\
\hline \multirow[t]{2}{*}{ feature } & $I_{\text {line }}\left(10^{-4} \mathrm{ph} \mathrm{cm}^{-2} \mathrm{~s}^{-1}\right)$ & $-1.2_{-0.8}^{+0.3}$ \\
\hline & $E W(\mathrm{eV})$ & $-33_{-20}^{+9}$ \\
\hline Fe XXVI & $E_{\text {line }}(\mathrm{keV})$ & $6.90_{-0.01}^{+0.02}$ \\
\hline absorption & $\sigma(\mathrm{keV})$ & $<0.043$ \\
\hline \multirow[t]{3}{*}{ feature } & $I_{\text {line }}\left(10^{-4} \mathrm{ph} \mathrm{cm}^{-2} \mathrm{~s}^{-1}\right)$ & $-1.4_{-0.4}^{+0.3}$ \\
\hline & $E W(\mathrm{eV})$ & $-42_{-13}^{+8}$ \\
\hline & $\chi^{2} /$ d.o.f. & $227.1 / 248$ \\
\hline
\end{tabular}

the cutoff power-law and in the COMPST models results in significantly better fits at a confidence level of $>99.99 \%$. In contrast, neither low-energy component improved the fit with the COMPTT model and so the best-fit with this model gives a $\chi^{2}$ of 264.3 for 249 d.o.f. The best-fit consists of absorbed blackbody and cutoff power-law continuum components and is shown in Fig. 2. The best fit parameter values, given in Table 2, consist of a blackbody with temperature, $k T_{\mathrm{bb}}$, of $1.30_{-0.06}^{+0.01} \mathrm{keV}$, and a cutoff power-law with a photon index, $\alpha$, of $1.90 \pm 0.02$ and a high energy cutoff, $E_{\mathrm{c}}$, of $15_{-4}^{+29} \mathrm{keV}$ for a $\chi^{2}$ of 227.1 for 248 d.o.f. This model has been used to model the spectra of a number of dipping LMXRB (e.g., Church et al. 1997), allowing an easy comparison with results from other sources. For comparison, if the blackbody is replaced by a disk-blackbody with a temperature of $2.12_{-0.03}^{+0.01} \mathrm{keV}$ and a projected inner disk radius of $2.20_{-0.03}^{+0.05} \mathrm{~km}$, the $\chi^{2}$ is 252.4 for 248 d.o.f. The best-fit $N_{\mathrm{H}}$ of $\left(3.5_{-0.1}^{+0.2}\right) 10^{21}$ atom $\mathrm{cm}^{-2}$ is a factor $\sim 2$ higher than the galactic column of $1.8 \times 10^{21}$ atom $\mathrm{cm}^{-2}$ (Dickey \& Lockman 1990), suggesting the presence of additional absorption. 


\subsection{Line spectral features}

The two narrow absorption features found in the persistent emission PN spectrum have energies of $6.64 \pm 0.02 \mathrm{keV}$ and $6.90_{-0.01}^{+0.02} \mathrm{keV}$, similar to the energies of the Fe xxv and FexXVI 1s-2p transitions which occur at $6.70 \mathrm{keV}$ and $6.97 \mathrm{keV}$. These correspond to the He- and H-like ionization states of $\mathrm{Fe}$ and are present over a wide range of temperatures and values of the ionization parameter, $\xi\left(\equiv L / n_{\mathrm{e}} r^{2}\right.$, where $L$ is the ionizing luminosity, $n_{\mathrm{e}}$ is the electron density and $r$ is the distance from the source). The measured offset of $\sim 60 \mathrm{eV}$ is consistent with the current energy calibration uncertainty of the PN small window mode (F. Haberl, private communication). When the instrumental broadening is removed the upper-limits to the line widths correspond to velocities of $<3600 \mathrm{~km} \mathrm{~s}^{-1}$ and $<2300 \mathrm{~km} \mathrm{~s}^{-1}$ for the Fe XXV and Fe XXVI transitions, respectively. We have searched for absorption edges due to Fe XXV and Fe XXVI at 8.83 and $9.28 \mathrm{keV}$ which might be expected due to the presence of absorption lines from these ions. However, there is no evidence for such features, but we caution that this is a spectral region where there is significant uncertainty in the PN calibration.

In order to search for any orbital dependence of the absorption features, five PN spectra covering orbital phases between 0.05 and 0.96 (where center of eclipse is phase $0.0)$ were extracted. These cover intervals of persistent emission and dipping activity (phases 0.65-0.90). The spectra were processed in the same way as the persistent emission spectrum discussed in Sect. 3.2. The residuals to the persistent emission best-fit (excluding the line features), are shown in Fig. 3. The depths of the features appear to be variable, but there is no obvious dependence of orbital phase (see Fig. 4), including during the interval of dipping activity. This means that similar amounts of the material responsible for this obscuration is present in the line of sight throughout most, or all, of the orbital cycle.

A similar analysis is not possible for the broad emission feature at $6.47 \mathrm{keV}$ due to its faintness. Assuming that the same energy offset $(60 \mathrm{eV})$ should be applied to the emission feature as with the absorption features, gives an energy of $6.53_{-0.14}^{+0.18} \mathrm{keV}$. Thus, the energy is consistent with $\mathrm{K} \alpha$ emission from a wide range of ionization states between neutral and He-like Fe. Given the broad line width $\left(\sigma=0.60_{-0.16}^{+0.12} \mathrm{keV}\right)$ it is likely that this feature originates from fluorescent excitation of $\mathrm{Fe}$ with a range of ionization states.

Oosterbroek et al. (2001) report the possible detection of a narrow emission line at $1.06 \pm 0.08 \mathrm{keV}$ with an $E W$ of $26 \pm 16 \mathrm{eV}$ in the BeppoSAX spectrum of MXB 1659-298 which they attribute to emission from Fe-L or Ne-K ions. A similar feature may be present at $94 \%$ confidence in the EPIC spectrum and is visible in the residuals of Fig. 2. Adding such a narrow feature to the best-fit model given in Table 2 gives an energy of $0.99 \pm 0.06 \mathrm{keV}$ and an $E W$ of $12_{-8}^{+6} \mathrm{eV}$.

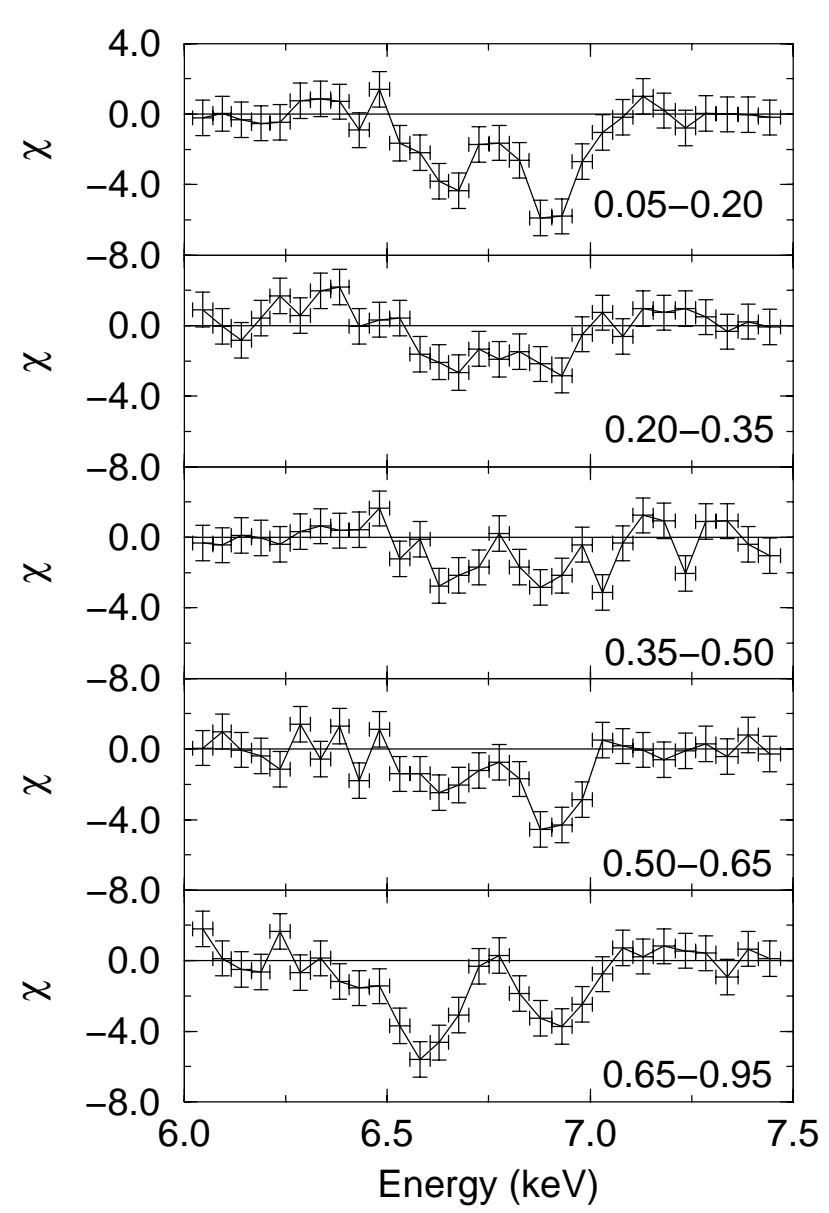

Fig. 3. Fit residuals with respect to the average persistent emission best-fit model (see Table 2) when the normalizations of the two narrow Fe absorption features seen in the PN are set to zero for different orbital phase (indicated).

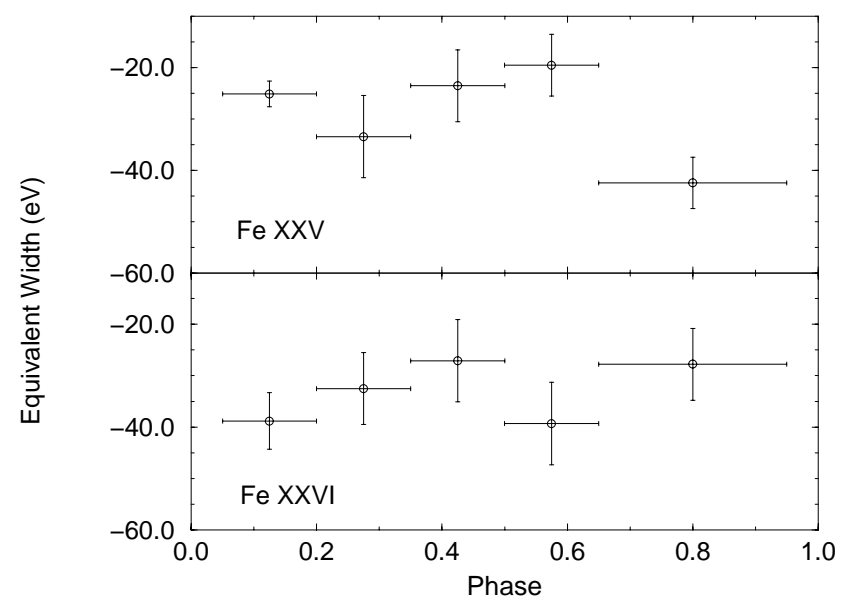

Fig. 4. The $E W \mathrm{~s}$ of the two Fe absorption features as a function of orbital phase. There is no obvious orbital dependence, even during the dipping activity at phase $\sim 0.8$.

\subsection{Eclipse spectrum}

As Fig. 1 indicates, there is significant flux remaining during the eclipses. Counts extracted from both the eclipses give a background subtracted $\mathrm{PN}$ rate of $5.29 \pm 0.09 \mathrm{~s}^{-1}$ for an exposure of $0.98 \mathrm{ks}$. With this low count rate it is 


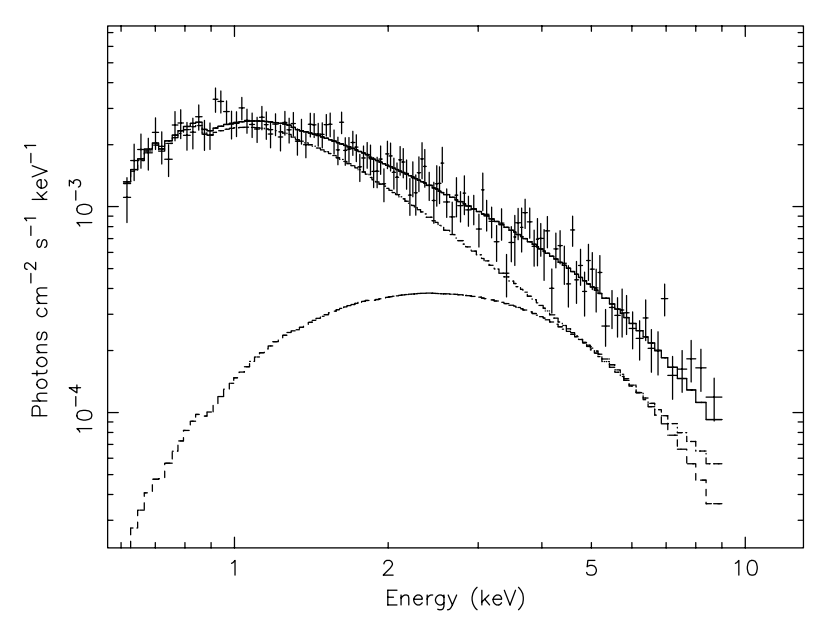

Fig. 5. The PN eclipse spectrum of MXB 1659-298 and the best-fit using the persistent emission spectrum consisting of a cutoff power-law and a blackbody. The spectral parameters were fixed at the values given in Table 2, except for the normalizations.

not necessary to exclude the core of the PSF, as for the persistent emission.

The eclipse spectrum can be well fit by the same continuum model as for the persistent emission with all the parameters fixed at the best-fit values given in Table 2, except for the normalizations of the two continuum components to give a $\chi^{2}$ of 95.6 for 125 d.o.f. (Fig. 5). Thismodel gives a 2-10 keV absorption corrected flux of $2.3 \times 10^{-11} \mathrm{erg} \mathrm{cm}^{-2} \mathrm{~s}^{-1}$ which corresponds to a $2-10 \mathrm{keV}$ luminosity of $5.8 \times 10^{35} \mathrm{erg} \mathrm{s}^{-1}$ at a distance of $15 \mathrm{kpc}$. This is $3.6 \%$ of the persistent emission. The $90 \%$ confidence upper-limits to the $E W$ s of the features at $6.47 \mathrm{keV}$, $6.64 \mathrm{keV}$ and $6.90 \mathrm{keV}$ are $380 \mathrm{eV},-121 \mathrm{eV}$ and $-42 \mathrm{eV}$, respectively, consistent with the persistent values.

\subsection{Dip spectrum}

In order to study the MXB 1659-298 spectrum during dipping intervals, 3 intensity selected spectra were extracted from intervals that excluded persistent emission and eclipses. Intervals corresponding to $\mathrm{PN} 0.5-10 \mathrm{keV}$ count rates (see Fig. 1) of $<10 \mathrm{~s}^{-1}$ ("deep dips"), 10 $40 \mathrm{~s}^{-1}$ ("medium dips") and 40-70 $\mathrm{s}^{-1}$ ("shallow dips") were extracted, with a time binning of $32 \mathrm{~s}$. The resulting background subtracted $\mathrm{PN}$ count rates are $7.21 \pm 0.09 \mathrm{~s}^{-1}$, $14.7 \pm 0.1 \mathrm{~s}^{-1}$, and $50.3 \pm 0.3 \mathrm{~s}^{-1}$ with exposure times of $1.0 \mathrm{ks}, 1.5 \mathrm{ks}$ and $0.5 \mathrm{ks}$, respectively. The core of the PSF was only excluded from the medium and shallow dip spectra, since the low count rate allowed the use of the entire PSF during the deep dips without significant pile-up being present.

In order to model the spectral changes during the MXB 1659-298 dips, we used the "complex continuum" approach of Church et al. (1997) which has been used to model the spectral changes observed from a number of dipping LMXRB. Following Church et al. (1997), the shapes and normalizations of both continuum components were held fixed at their persistent emission values.
Table 3. Best-fit spectral parameters for the 3 dipping intervals. The continuum model used is a cutoff power-law and a blackbody to which three Gaussian lines are added. Only the parameters for the partial covering (PCFABS) models used to represent the additional absorption during dips are given. $N_{\mathrm{H}}$ is in units of $10^{22}$ atom $\mathrm{cm}^{-2}$.

\begin{tabular}{lccc}
\hline Parameter & \multicolumn{3}{c}{ Dip Spectrum } \\
& Shallow & Medium & Deep \\
\hline$N_{\mathrm{H}_{\mathrm{bb}}}$ & $25_{-8}^{+10}$ & $49_{-6}^{+7}$ & $157_{-38}^{+63}$ \\
$f_{\mathrm{bb}}$ & $0.74 \pm 0.09$ & $0.67 \pm 0.02$ & $0.88 \pm 0.01$ \\
$N_{\mathrm{H}_{\mathrm{cpl}}}$ & $1.4_{-0.2}^{+0.1}$ & $14 \pm 1$ & $52_{-3}^{+6}$ \\
$f_{\mathrm{cpl}}$ & $0.75 \pm 0.01$ & $0.968_{-0.001}^{+0.002}$ & $0.960 \pm 0.002$ \\
$\chi^{2} /$ d.o.f. & $276.4 / 203$ & $241.6 / 225$ & $262.2 / 190$ \\
\hline
\end{tabular}

In addition to a galactic column, $N_{\text {gal }}$, which was fixed at $1.8 \times 10^{21}$ atom $\mathrm{cm}^{-2}$ (Dickey \& Lockman 1990), the cutoff power-law was obscured by an additional partial covering component during the dips. The three line features seen in the persistent emission by the PN were also included in the model. This gave unacceptable fits with $\chi^{2}$ values of 310.3 for 204 d.o.f. (shallow dips), 624.7 for 226 d.o.f. (medium dips) and 564.7 for 191 d.o.f. (deep dips). Inspection of the residuals shows that a component with a shape similar to that of the blackbody is still visible at low-energies, so partial covering was added to this component as well. The intensity during the dips is then modeled as:

$$
\begin{aligned}
& \mathrm{e}^{-\sigma_{\mathrm{MM}} N_{\mathrm{gal}}}\left[\left[f_{\mathrm{bb}} \mathrm{e}^{-\sigma_{\mathrm{MM}} N_{\mathrm{H}_{\mathrm{bb}}}}+\left(1-f_{\mathrm{bb}}\right)\right] I_{\mathrm{BB}}\right. \\
& \left.\quad+\left[f_{\mathrm{cpl}} \mathrm{e}^{-\sigma_{\mathrm{MM}} N_{\mathrm{H}_{\mathrm{cpl}}}}+\left(1-f_{\mathrm{cpl}}\right)\right] I_{\mathrm{CPL}}+I_{\mathrm{G}_{1}}+I_{\mathrm{G}_{2}}+I_{\mathrm{G}_{3}}\right],
\end{aligned}
$$

where $N_{\mathrm{H}_{\mathrm{bb}}}$ and $N_{\mathrm{H}_{\mathrm{cpl}}}$ are the additional absorptions for the continuum spectral components and $f_{\mathrm{bb}}$ and $f_{\mathrm{cpl}}$ are their covering fractions $(0<f<1) ; I_{\mathrm{BB}}, I_{\mathrm{CPL}}$ and $I_{\mathrm{G}_{\mathrm{i}}}$ are the normalizations of the blackbody, cutoff powerlaw and the three Gaussian lines, respectively. Thus, the only free parameters in the fitting are $N_{\mathrm{H}_{\mathrm{bb}}}, N_{\mathrm{H}_{\mathrm{cpl}}}, f_{\mathrm{bb}}$ and $f_{\mathrm{cpl}}$. The fits are significantly better with $\chi^{2}$ values of 276.4 for 203 d.o.f., 241.6 for 225 d.o.f., and 262.2 for 190 d.o.f. for the shallow, medium and deep dipping spectra, respectively (Table 3 ). The blackbody suffers significantly more absorption than the cutoff power-law $\left(\sim 1.5 \times 10^{24}\right.$ atom $\mathrm{cm}^{-2}$ compared to $\sim 5 \times 10^{23}{\text { atom } \mathrm{cm}^{-2}}^{-2}$ during the deepest dips), but has a smaller covering fraction. Whilst the fit to the medium dipping spectrum is reasonably good, the fits to the other two dip spectra are formally unacceptable and so the results should be treated with caution.

Inspection of the fit residuals revealed structured positive residuals around 6-7 keV. Better fits are obtained if a partial covering absorber, with an additional column of $N_{\mathrm{H}_{\text {gau }}}$ and partial covering fraction $f_{\text {gau }}$ affects the $\mathrm{Fe}$ emission. If these parameters are set to the values derived for the cutoff power-law, then the $\chi^{2}$ values reduce significantly in the case of the medium dipping interval. Smaller reductions in $\chi^{2}$ are evident for the deep and shallow dip 
Table 4. Line features visible in the RGS persistent emission spectrum. $E W$ is the line equivalent width. The wavelength uncertainties do not include a systematic uncertainty of $0.01 \AA$. The line widths are $1 \sigma$ upper-limits. The line widths expressed in $\mathrm{km} \mathrm{s}^{-1}$ and $\mathrm{keV}$ are after removal of the instrumental broadening. Theoretical wavelengths are from Mewe et al. (1985).

\begin{tabular}{lccccccc}
\hline $\begin{array}{l}\text { Ion and } \\
\text { Transition }\end{array}$ & $\begin{array}{c}\text { Measured } \\
\text { Wavelength }(\AA)\end{array}$ & $\begin{array}{c}\text { Theoretical } \\
\text { Wavelength }(\AA)\end{array}$ & $\begin{array}{c}E W \\
(\mathrm{eV})\end{array}$ & $\begin{array}{c}\text { Line intensity } \\
\left(\mathrm{ph} \mathrm{cm}^{-2} \mathrm{~s}^{-1}\right)\end{array}$ & \multicolumn{2}{c}{$\begin{array}{c}\text { Line width }(\sigma) \\
(\AA)\end{array}$} & $\begin{array}{l}\left(\mathrm{km} \mathrm{s}^{-1}\right) \\
(\mathrm{keV})\end{array}$ \\
\hline O VIII 1s-2p & $18.95_{-0.01}^{+0.02}$ & 18.970 & $-2.6 \pm 0.4$ & $-(3.9 \pm 0.6) \times 10^{-5}$ & $<0.045$ & $<600$ & $<20$ \\
O VIII 1s-3p & $16.00_{-0.01}^{+0.02}$ & 16.010 & $-1.3 \pm 0.4$ & $-(3.2 \pm 1.1) \times 10^{-5}$ & $<0.070$ & $<1200$ & $<80$ \\
O VIII 1s-4p & $15.21 \pm 0.01$ & 15.180 & $-1.6 \pm 0.4$ & $-(4.5 \pm 1.1) \times 10^{-5}$ & $<0.058$ & $<1000$ & $<60$ \\
Ne X 1s-2p & $12.15_{-0.01}^{+0.02}$ & 12.130 & $-2.1 \pm 0.4$ & $-(1.0 \pm 0.2) \times 10^{-4}$ & $<0.049$ & $<1000$ & $<70$ \\
\hline
\end{tabular}
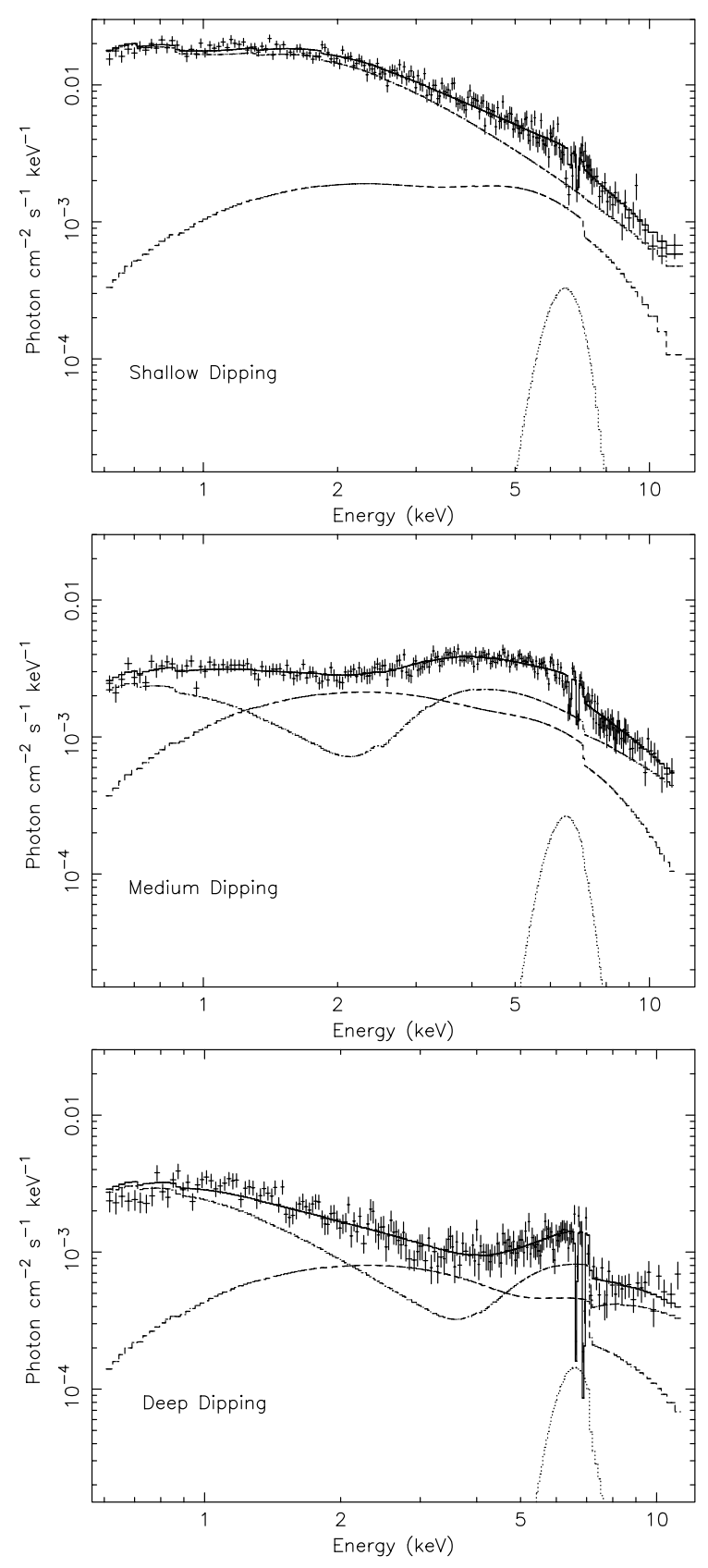

Fig. 6. EPIC PN $0.6-12 \mathrm{keV}$ dipping photon spectra deconvolved using the partial covering model with the parameters given in Table 3. The contributions of the cutoff power-law (which dominates at high energies), blackbody and line features are shown separately. spectra. This suggests that the iron line does indeed suffer extra absorption during dipping intervals, but that we are unable to conclude whether the absorbing material has similar properties as that responsible for the absorption of the blackbody or cutoff power-law components.

\section{RGS results}

\subsection{Absorption lines}

The first order RGS1 and RGS2 summed spectrum corresponding to the same persistent emission interval as used for the EPIC PN was extracted and is displayed in Fig. 7. The spectrum is dominated by a smooth continuum with a number of low-intensity features evident. There is no evidence for the He-like triplet of O VII or O VIII 1s-2p in emission as seen from EXO 0748-676 (Cottam et al. 2001). Instead, we detect 4 narrow absorption lines which we identify with Nex 1s-2p transition at $12.130 \AA$, O viII $1 \mathrm{~s}-2 \mathrm{p}$ at $18.970 \AA$, O vIII $1 \mathrm{~s}-3 \mathrm{p}$ at $16.010 \AA$ and O viII $1 \mathrm{~s}-4 \mathrm{p}$ transitions at $15.180 \AA$. These features are shown in Figs. 7 and 8 and their properties summarized in Table 4 . In addition, there are unidentified features near $23 \AA$ which may be instrumental in origin. The two 1s-2p features are clearly evident, while the other two O VIII transitions are less clear. Their measured wavelengths are consistent with the theoretical values when the uncertainty in the wavelength scale of the RGS (currently $0.008 \AA$, den Herder et al. 2001) is included (see Table 4). All the features are unresolved and their widths are compatible with the RGS line spread function of $\sim 0.06 \AA$. When the instrumental broadening has been subtracted, the upper-limits to the widths correspond to velocities of $<600 \mathrm{~km} \mathrm{~s}^{-1}$ and $<1000 \mathrm{~km} \mathrm{~s}^{-1}$ for the $\mathrm{O}$ and $\mathrm{Ne} 1 \mathrm{~s}-$ $2 \mathrm{p}$ transitions, respectively. Figure 8 shows the spectral regions around the lines in more detail.

RGS spectra during dipping intervals (orbital phases $0.65-0.95$ ) were extracted and processed in the same way as for the persistent emission spectra. The exposure time is $13 \mathrm{ks}$ and the mean count rate $0.72 \mathrm{~s}^{-1}$. Figure 7 shows the dip spectra together with those obtained during the persistent intervals. While the persistent spectra clearly show the two lines in absorption, these are not so evident in the dip spectra. However, fitting narrow Gaussians to the dip spectra gives upper-limits to the $E W \mathrm{~s}$ of $1.4 \pm 0.5$ and $1.5 \pm 1.0 \mathrm{eV}$ to any features at the wavelengths of the 


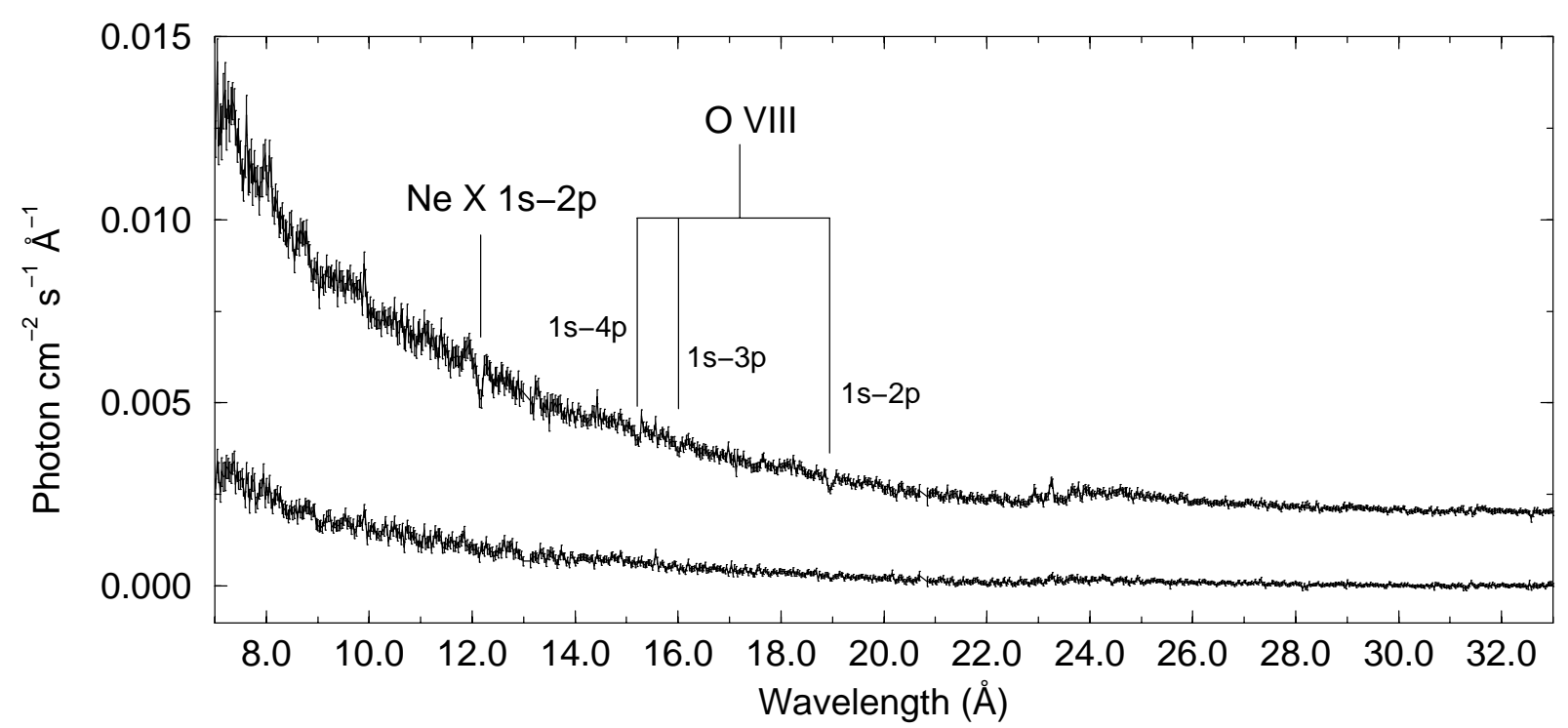

Fig. 7. The first order RGS spectra of MXB 1659-298 with a binning of $0.03 \AA$. The upper curve is the persistent emission spec-

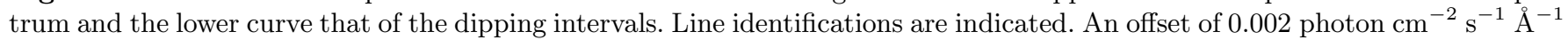
has been added to the persistent emission spectrum for clarity.
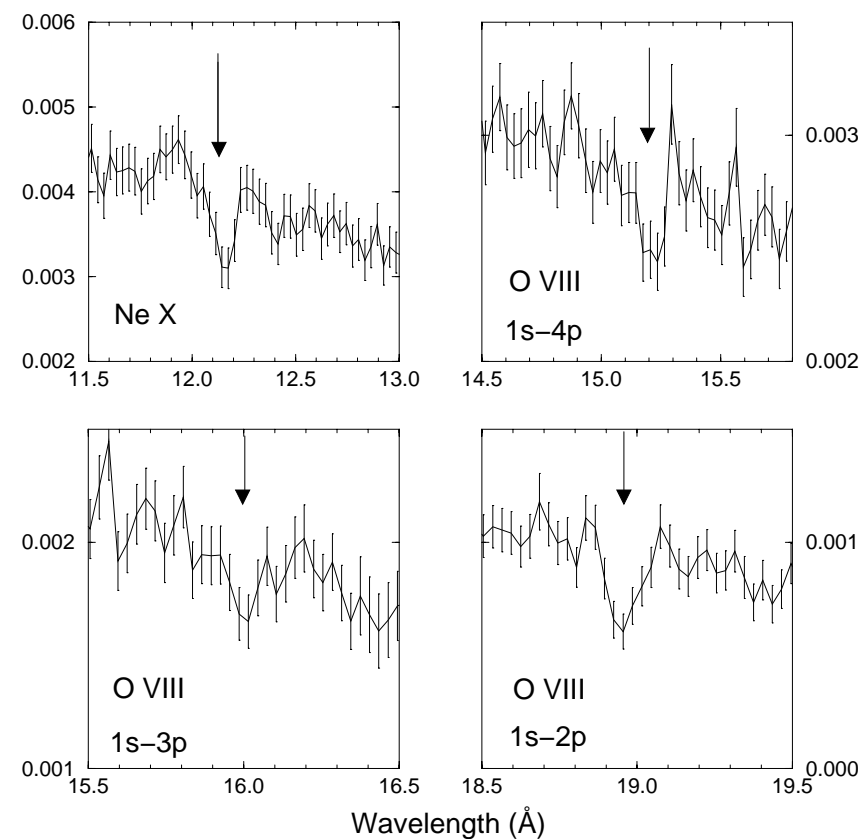

Fig. 8. Persistent emission RGS spectra in the regions around the narrow absorption features with a binning of $0.03 \AA$ in units of photon $\mathrm{cm}^{-2} \mathrm{~s}^{-1} \AA^{-1}$. Theoretical wavelengths are indicated.

O viII and Nex 1s-2p transitions. Thus, we cannot exclude the presence of these features during dipping intervals with the same $E W$ s as during the persistent emission.

\section{OM results}

A total of $20 B$ filter OM images were obtained. The counterpart to MXB 1659-298 (V2134 Oph) is clearly recognizable in the images and is significantly brighter than in the $I$-band image of Wachter et al. (2000). The magnitudes of V2134 Oph were obtained by integrating the counts in a circular region with a radius of $4^{\prime \prime}$ ( 8 pixels). This is slightly smaller than the recommended value of 12 pixels (Gondoin 2001), but was chosen because of crowding in the field. The background was determined from a region 0 '45 East of the source with a radius of 16 pixels. The background subtracted counts were converted to magnitudes using Star 1 which has a $B$-magnitude of 18.1 in the USNO A2.0 catalog. Due to the relatively poor OM spatial resolution and the large difference in brightness, the contribution of the faint star close to, and partially resolved from MXB 1659-298, is included in the magnitude determinations. The uncertainty in magnitude was estimated from a comparison of the derived Star 1 and 2 mag to be $\sim 2 \%$ or 0.02 mag.

Figure 9 shows the lightcurve together with the rebinned $0.5-10 \mathrm{keV}$ PN lightcurve. A sinusoid-like optical modulation is present, with a narrow minimum coincident with the X-ray eclipse superposed. The peak-to-peak amplitude is $\sim 0.5 \mathrm{mag}$ and a mean value $\sim 18.5 \mathrm{mag}$. The overall shape of the modulation is consistent with that reported in Wachter et al. (2000). The broad modulation of the optical lightcurve with a minimum coincident with the X-ray eclipse is consistent with the obscuration of an extended object, such as an accretion disk, centered on the compact object.

\section{Discussion}

We report the results of an XMM-Newton observation of the dipping, eclipsing, bursting LMXRB MXB 1659-298 in 2001 February. EPIC and RGS spectra of the persistent emission reveal the presence of narrow resonant absorption features identified with $\mathrm{O}$ VIII $1 \mathrm{~s}-2 \mathrm{p}, 1 \mathrm{~s}-3 \mathrm{p}$ and $1 \mathrm{~s}-4 \mathrm{p}$, Ne X 1s-2p, Fe XXV 1s-2p, and Fe XXVI 1s-2p transitions, together with a broad Fe emission feature at $6.47_{-0.14}^{+0.18} \mathrm{keV}$. This is in striking contrast to EXO 0748-676 where RGS observations revealed the presence of strong emission 


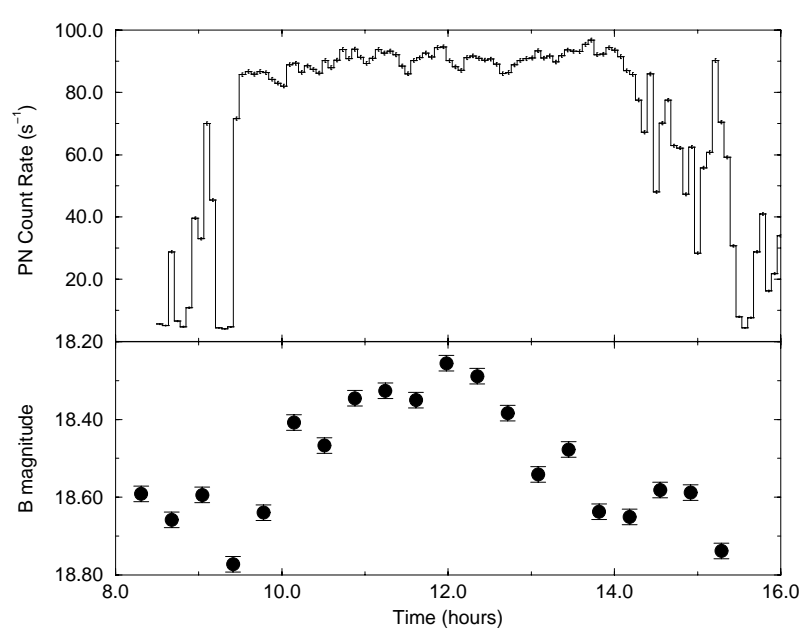

Fig. 9. EPIC PN (0.5-10 keV) and OM ( $B$ filter) lightcurves of MXB 1659-298. Time is hours of 2001 February 20. An X-ray eclipse is visible at 9:20 hr.

features from $\mathrm{O}$ VII and $\mathrm{O}$ VIII as well as weaker emission lines from Ne IX, Ne X and N VII and photo-electric absorption edges of both O VII and O VIII (Cottam et al. 2001). The $E W \mathrm{~s}$ of the $\mathrm{Fe}$ absorption features observed from MXB 1659-298 show no obvious dependence on orbital phase, even during dipping intervals. The $E W \mathrm{~s}$ of the $\mathrm{O}$ VIII and Ne x 1s-2p features are consistent with having the same values during persistent and dipping intervals. The absorption features are narrow with upper-limits to any widths as low as $<600 \mathrm{~km} \mathrm{~s}^{-1}$ (O VIII, $1 \mathrm{~s}-2 \mathrm{p}$ ). The overall shape of the $0.6-12 \mathrm{keV}$ continuum is similar to many other LMXRBs and may be modeled using blackbody and cutoff power-law components. During dips the blackbody suffers significantly more absorption than the cutoff power-law $\left(\sim 1.5 \times 10^{24}\right.$ atom $\mathrm{cm}^{-2}$ compared to $\sim 5 \times 10^{23}$ atom $\mathrm{cm}^{-2}$ during the deepest dips). Similar behavior has been observed from other LMXRBs (e.g., XB 1916-053, Church et al. 1997), but is in contrast to recent XMM-Newton EPIC results from EXO 0748-676 (Bonnet-Bidaud et al. 2001) and BeppoSAX results from Oosterbroek et al. (2001) where the hard components in these sources are seen to undergo strong absorption during dips. The BeppoSAX result is especially intriguing since it is from the same source, with the same quoted $2-10 \mathrm{keV}$ luminosity $\left(1.6 \times 10^{37} \mathrm{erg} \mathrm{s}^{-1}\right)$, and from an observation performed only 6 months earlier during the same outburst. This change in dipping properties, if confirmed, suggests that the location, size and structure of the emitting regions in LMXRBs can change on a timescale of months without any apparent change in the X-ray luminosity.

Narrow X-ray absorption lines have been detected from the superluminal jet sources GRO J1655-40 (Ueda et al. 1998; Yamaoka et al. 2001) and GRS 1915+105 (Kotani et al. 2000; Lee et al. 2001). ASCA observations of GRO J1655-40 revealed the presence of absorption features due to Fe XXV and Fe XXVI which, like those observed here, do not show any obvious dependence of their $E W \mathrm{~s}$ on orbital phase. GRO J1655-40 has been observed

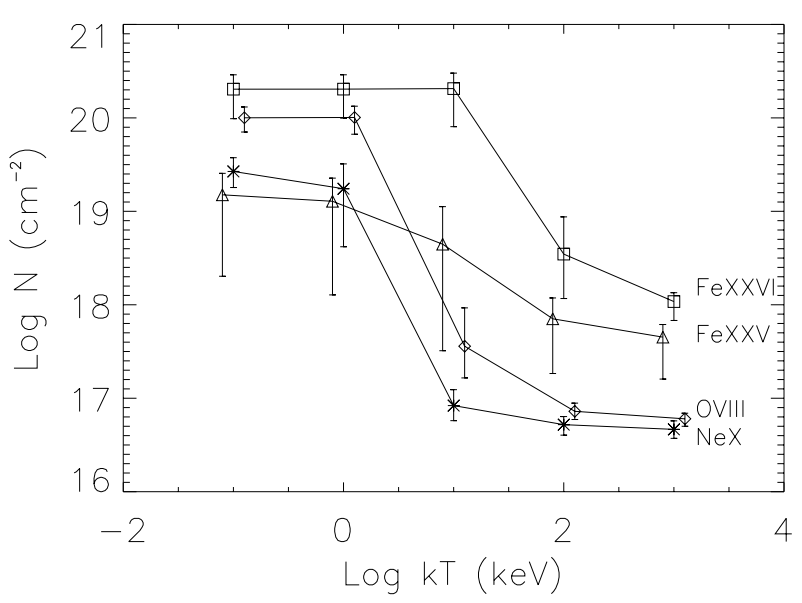

Fig. 10. Ion column densities derived from the curve of growth analysis for the O VIII 1s-2p, Ne x, Fe XXV and Fe XXVI absorption lines. The curves have been offset slightly along the temperature axis for clarity.

to undergo deep absorption dips (Kuulkers et al. 1998) consistent with observing the source at a high inclination of $60^{\circ}-75^{\circ}$ (e.g., Frank et al. 1987). ASCA observations of GRS 1915+105 revealed, in addition, absorption features due to $\mathrm{Ca}$ Xx, Ni XXVII and Ni XXVIII. A recent Chandra HETGS observation of this source showed broad $\left(\sim 1000 \mathrm{~km} \mathrm{~s}^{-1}\right)$ absorption features from Fe XXV and Fe XxVI. Until now, it was possible that these absorption features are peculiar to superluminal jet sources and related in some way to the jet formation mechanism. With the discovery of absorption features from the LMXRB MXB 1659-298 this appears not to be the case, and as proposed by Kotani et al. (2000) ionized absorption features may be common characteristics of accreting systems which are viewed close to edge-on. The discovery of these features from the two superluminal sources may simply be a selection effect due to their high fluxes ( $\sim 1 \mathrm{Crab})$ compared to other systems.

In the likely hypothesis of photo-ionization (e.g., Bautista et al. 1998), the presence of O VIII and Ne X ions is indicative of conditions where $\xi$ is $\sim 100 \mathrm{erg} \mathrm{cm} \mathrm{s}^{-1}$, whereas Fe XXV and Fe XXVI are present in regions where $\xi \gtrsim 1000 \mathrm{erg} \mathrm{cm} \mathrm{s}^{-1}$ (Kallman \& McCray 1982). This broad range of $\xi$ suggests either that the absorbing material is present over a wide range of distances from the central source, or has a large range of densities. The absorption features observed from MXB 1659-298 are present during a wide range of orbital phases. This excludes the possibility that the absorbing material is associated with that responsible for the dipping activity centered on phase $\sim 0.8$. As pointed out by Ueda et al. (1998) the plasma responsible for the absorption features must be an-isotropically located, or at least illuminated, around the central X-ray source, otherwise emission from outside the line of sight would completely fill in the absorption features.

In order to estimate the column densities of $\mathrm{O}, \mathrm{Ne}$ and Fe that produced the observed absorption lines we 
performed a curve of growth analysis following that of Kotani et al. (2000) for GRS $1915+105$. This allows the $E W \mathrm{~s}$ of the lines to be converted into column densities depending on the kinematic temperature of the absorbing material. The kinematic temperature includes contributions from thermal motions as well as any bulk motions. Absorbing columns for the O VIII 1s-2p, Ne X, Fe XXV and $\mathrm{Fe}$ XXVI features were derived for a range of assumed temperatures (Fig. 10). As expected, a lower kinematic temperature requires a higher ion column density, and hence a higher hydrogen column density. However the $N_{\mathrm{H}}$ should not exceed one Thomson optical depth $\left(1.5 \times 10^{24}\right.$ atom $\left.\mathrm{cm}^{-2}\right)$ otherwise the absorption lines would be strongly diminished. We assume abundances of $8.5 \times 10^{-4}, 1.2 \times 10^{-4}$ and $4.7 \times 10^{-5}$ for $\mathrm{O}, \mathrm{Ne}$, and $\mathrm{Fe}$, respectively (Anders \& Grevesse 1989). Examination of Fig. 10 shows that the total Fe column density must be $\lesssim 7 \times 10^{19} \mathrm{~cm}^{-2}$ for $N_{\mathrm{H}}$ to be less than one Thomson depth. This implies that $k T_{\mathrm{Fe}} \gtrsim 25 \mathrm{keV}$. A further constraint on the kinematic temperature of the absorbing plasma can be obtained from the upper-limits to the widths of the lines. In the case of the Fe Xxv and FexxvI lines these are not very constraining $(<2400 \mathrm{keV}$ and $<1000 \mathrm{keV}$, respectively), whilst those for the other lines, derived using Eq. (1) of Yamaoka et al. (2001), are given in Table 4. These show that $k T_{\mathrm{Fe}}$ is inconsistent with the upper-limit obtained from the $\mathrm{O}$ VIII $1 \mathrm{~s}-2 \mathrm{p}$ transition of $<20 \mathrm{keV}$ which implies that the $\mathrm{O}$ column density is $>3 \times 10^{17} \mathrm{~cm}^{-2}$. This again suggests that plasma with a range of conditions is responsible for the absorption features. Indeed, at a temperature of $25 \mathrm{keV} \gtrsim 50 \%$ of the $\mathrm{Fe}$ will be fully ionized (e.g., Arnaud \& Rothenflug 1985) as will virtually all of the lower $Z$ elements. This probably explains why the source is not blanketed by the extremely high absorption implied by the Fe lines - most of the material that could photo-electrically absorb at lower energies is fully ionized. Indeed, even assuming an extremely high kinematic temperature of $1000 \mathrm{keV}$, the $N_{\mathrm{H}}$ derived from the Fe absorption features is $2 \times 10^{22}$ atom $\mathrm{cm}^{-2}$, a factor of 10 higher than the excess low-energy neutral absorption measured during quiescent intervals (Table 2).

Following Yamaoka et al. (2001) and assuming that the plasma is in hydro-dynamical equilibrium in the direction vertical to the disk plane, the distance, $r$, of the absorbing plasma from the central source can be estimated from its thermal temperature using $(h / r)^{2} \times\left(G M m_{\mathrm{H}} / r\right)=k T_{\mathrm{th}}$ (e.g., Shakura \& Sunyaev 1973). Here $M$ is the neutron star mass, $G$ the gravitational constant, $m_{\mathrm{H}}$ the mass of a hydrogen atom and $h$ the scale height of the plasma. Assuming that $h / r=\tan \left(90^{\circ}-i\right)$, where $i$ is the binary inclination angle $\left(i \sim 80^{\circ}\right)$ gives $r_{\mathrm{Fe}} \lesssim 2.4 \times 10^{8} \mathrm{~cm}$ for $k T_{\mathrm{Fe}} \gtrsim 25 \mathrm{keV}$. Similarly, the lower limits to $r$ derived from the temperatures given in Table 4 are $r_{\mathrm{O}} \gtrsim 3 \times$ $10^{8} \mathrm{~cm}$ and $r_{\mathrm{Ne}} \gtrsim 9 \times 10^{7} \mathrm{~cm}$. For a companion star mass of $0.5 M_{\odot}$ the binary separation is $2 \times 10^{11} \mathrm{~cm}$ and the radius of the accretion disk (assumed to be $=0.8 \times R_{\mathrm{L}}$, where $R_{\mathrm{L}}$ is the Roche Lobe radius of the neutron star) is $5 \times 10^{10} \mathrm{~cm}$. Thus, the material responsible for the $\mathrm{Fe}$ absorption must be located close to the central source, at a radius $\sim 200$ times less than that of the accretion disk, while the material responsible for the $\mathrm{O}$ and Ne absorption is located at larger radii, consistent with the lower value of the ionization parameter for these elements. Using the ionization parameter values ( $\sim 100$ for oxygen and neon; $>1000$ for iron), and the distance from the central source, $r$, calculated before, it is possible to roughly estimate the electron density, $n_{\mathrm{e}}$ : we find $n_{\mathrm{e}} \sim 2 \times 10^{18} \mathrm{~cm}^{-3}$ from the $\mathrm{O}$ absorption lines, $n_{\mathrm{e}} \sim 2 \times 10^{19} \mathrm{~cm}^{-3}$ from Ne and $n_{\mathrm{e}} \sim 3 \times 10^{17} \mathrm{~cm}^{-3}$ from Fe. Assuming that $N_{\mathrm{H}}=n_{\mathrm{e}} \Delta r$, where $\Delta r$ is the thickness of the absorbing layer, we derive $\Delta r \sim 200-4 \times 10^{4} \mathrm{~cm}$ for $\mathrm{O}, \Delta r \sim 30-3 \times 10^{4} \mathrm{~cm}$ for Ne and $\Delta r<5 \times 10^{6} \mathrm{~cm}$ for Fe.

Since the absorption features occur through a wide range of orbital phases, the absorbing material is most likely located in a cylindrical geometry with an axis perpendicular to that of the accretion disk. This geometry could explain why similar absorption features have not been so far observed from other LMXRBs that are viewed at angles further from the orbital plane.

Note added in proof: After acceptance of the paper we became aware of the discovery of an absorption feature at $7 \mathrm{keV}$ (in terpreted as absorption from FeXXVI ions) with ASCA in the LMXRB GX13+1 by Ueda Y., Asai K., Yamaoka K., Donati T. \& Inoue H. (2001, ApJ, 556, L87). This detection in a neutron star binary rules out that these spectral features are unique to the Galactic superluminal jet sources.

Acknowledgements. The results presented are based on observations obtained with XMM-Newton, an ESA science mission with instruments and contributions directly financed by ESA Member States and the USA (NASA). L. Sidoli acknowledges an ESA Research Fellowship and thanks A. Borriello, S. Molendi and A. Orr for helpful discussions. We thank G. Vacanti and F. Haberl for assistance and T. Kotani for making his curve of growth software available.

\section{References}

Anders, E., \& Grevesse, N. 1989, Geochim. Cosmochim. Acta, 53,197

Arnaud, M., \& Rothenflug, R. 1985, A\&AS, 60, 425

Augusteijn, T., Freyhammer, L., \& in 't Zand, J. 1999, IAU Circ., 7138

Bautista, M. A., Kallman, T. R., Angelini, L., et al. 1998, ApJ, 509,848

Bonnet-Bidaud, J. M., Haberl, F., Ferrando, F., Bennie, P. J., \& Kendziorra, E. 2001, A\&A, 365, L282

Church, M. J., \& Bałucińska-Church, M. 1995, A\&A, 300, 441

Church, M. J., Dotani, T., Bałucińska-Church, M., et al. 1997, ApJ, 491, 388

Cominsky, L. R. 1981, Ph.D. Thesis, MIT

Cominsky, L. R., \& Wood, K. S. 1984, ApJ, 283, 765

Cominsky, L. R., \& Wood, K. S. 1989, ApJ, 337, 485

Cottam, J., Kahn, S. M., Brinkman, A. C., den Herder, J. W., \& Erd, C. 2000, A\&A, 365, L277

Den Herder, J. W., Brinkman, A. C., Kahn, S. M., et al. 2001, A\&A, 365, L7 
Dickey, J. M., \& Lockman, F. J. 1990, ARA\&A, 28, 215

Doxsey, R., Grindlay, J., Griffiths, R., et al. 1979, ApJ, 228, L67

Frank, J., King, A. R., \& Lasota, J.-P. 1987, A\&A, 178, 137

Gondoin, Ph. 2001, User's Guide of the XMM-Newton Science Analysis System, Technical Report XMM-PS-GM-21 http://xmm.vilspa.esa.es/public/xmm_sas_sv_top.html

In 't Zand, J. J. M., Heise, J., Smith, M. J. S., et al. 1999, IAU Circ., 7138

Jansen, F., Lumb, D., Altieri, B., et al. 2001, A\&A, 365

Kallman, T. R., \& McCray, R. 1982, ApSS, 50, 263

Kotani, T., Ebisawa, K., Dotani, T., et al. 2000, ApJ, 539, 413

Kuulkers, E., Wijnards, R., Belloni, T., et al. 1998, ApJ, 494, 753

Lee, J. C., Schulz, N. S., Reynolds, C. S., Fabian, A. C., \& Blackman, E. G. 2001, Proc. of X-ray Astronomy 2000 [astro-ph/0012111]

Lewin, W. H. G., Hoffmann, J. A., Doty, J., \& Liller, W. 1976, IAU Circ., 2994, 2

Makishima, K., Maejima, Y., Mitsuda, K., et al. 1986, ApJ, 285,712
Mason, K. O., Breeveld, A., Much, R., et al. 2001, A\&A, 365, L36

Mewe, R., Gronenschild, E. H. B. M., \& van den Oord, G. H. J. 1985, A\&AS, 62, 197

Mitsuda, K., Inoue, H., Koyama, K., et al. 1984, PASJ, 36, 741

Morrison, D., \& McCammon, D. 1983, ApJ, 270, 119

Oosterbroek, T., Parmar, A. N., Sidoli, L., in' t Zand, J. J. M., \& Heise, J. 2001, A\&A, 376, 532

Parmar, A. N., White, N. E., Giommi, P., \& Gottwald, M. 1986, ApJ, 308, 199

Shakura, N. I., \& Sunyaev, R. A. 1973, A\&A, 24, 337

Strüder, L., Briel, U., Dennerl, K., et al. 2001, A\&A, 365, L18

Titarchuk, L. 1994, ApJ, 434, 570

Turner, M. J. L., Abbey, A., Arnaud, M., et al. 2001, A\&A, $365, \mathrm{~L} 27$

Ueda, Y., Inoue, H., Tanaka, Y., et al. 1998, ApJ, 492, 782

Yamaoka, K., Ueda, Y., Inoue, H., et al. 2001, PASJ, 53, 179

Wachter, S., Smale, A. P., \& Bailyn, C. 2000, ApJ, 534, 367 